Supporting Information for:

\title{
Double Helix-to-Double Helix Transformation Using Platinum(II) Acetylide Complexes as Surrogate Linkers
}

\author{
Yoshio Furusho, ${ }^{*, \dagger}$ Yoshie Tanaka, ${ }^{\dagger}$ and Eiji Yashima ${ }^{*,+, \neq}$
}

Yashima Super-structured Helix Project, Exploratory Research for Advanced Technology (ERATO), Japan Science and Technology Agency (JST), Creation Core Nagoya 101, 2266-22 Anagahora, Shimoshidami, Moriyama-ku, Nagoya 463-0003, Japan, and Institute for Advanced Research, Nagoya University, Nagoya 464-8601, Japan

E-mail: furusho@yp-jst.jp; yashima@apchem.nagoya-u.ac.jp

\section{Table of Contents}

1. Materials

2. Instruments

3. Synthetic Procedures

4. Characterization of the trans-Pt(II)- $\mathrm{PPh}_{3}$ Duplex $\mathbf{3 a} \cdot \mathbf{4 a}$

${ }^{1} \mathrm{H}$ NMR $\left(500 \mathrm{MHz}, \mathrm{CDCl}_{3}, 25{ }^{\circ} \mathrm{C}\right)$ Spectra of $(R)-\mathbf{3 a}$ and $(R)-\mathbf{3 a} \cdot 4 \mathbf{a}$

ESI-MS Spectrum of $(R)-\mathbf{3 a} \cdot \mathbf{4 a}$

5. Characterization of the cis-Pt(II)-dppee Duplex $\mathbf{3 b} \cdot \mathbf{4 b}$

${ }^{1} \mathrm{H}$ NMR (500 MHz, $\left.\mathrm{CDCl}_{3}, 25{ }^{\circ} \mathrm{C}\right)$ Spectra of $(R)-\mathbf{3 b}$ and $(R)-\mathbf{3 b} \cdot \mathbf{4 b}$

ESI-MS Spectrum of $(R)-\mathbf{3 b} \cdot \mathbf{4 b}$

6. ${ }^{31} \mathrm{P}$ NMR (200 MHz, $\left.\mathrm{CDCl}_{3}, 25^{\circ} \mathrm{C}\right)$ Spectral Data

7. Optical Rotations

8. Structural Transformation of the Duplexes

$(R)-\mathbf{3 a} \cdot \mathbf{4 a}$ to $(R)-\mathbf{3 b} \cdot \mathbf{4 b}$

$(R)-\mathbf{3 b} \cdot \mathbf{4 b}$ to $(R)-\mathbf{1} \cdot \mathbf{2}$

9. MM-Calculated Structures of the Duplexes $\mathbf{3 a} \cdot \mathbf{4} \mathbf{a}$ and $\mathbf{3 b} \cdot \mathbf{4 b}$ 


\section{Materials}

All starting materials were purchased from commercial suppliers and were used without further purification unless otherwise noted. The chiral amidines $(R)-$ and $(S)-\mathbf{5}$ and the achiral carboxylic acid $\mathbf{6}$ were prepared according to literature methods. ${ }^{1}$

\section{Instruments}

The NMR spectra were measured using a Varian UNITY INOVA 500AS spectrometer operating at $500 \mathrm{MHz}$ for ${ }^{1} \mathrm{H}$, at $125 \mathrm{MHz}$ for ${ }^{13} \mathrm{C}$, and at $200 \mathrm{MHz}$ for ${ }^{31} \mathrm{P}$. The IR spectra were recorded using a Jasco Fourier Transform IR-680 Plus spectrophotometer. The absorption and CD spectra were measured in a 1-mm quartz cell on a Jasco V-570 spectrophotometer and a Jasco J-820 spectropolarimeter, respectively. Optical rotation was measured in a 5.0-cm quartz cell on a Jasco P-1030 polarimeter. Elemental analyses were performed by the Nagoya University Analytical Laboratory in the Graduate School of Engineering. The electrospray ionization mass (ESI-MS) spectra were recorded using a JEOL JMS-T100CS mass spectrometer. The molecular modeling and molecular mechanics were performed using the Forcite software and the Universal Force Field in the Materials Studio package (version 3.0; Accerlys Inc.).

1. Tanaka, Y.; Katagiri, H.; Furusho, Y.; Yashima, E. Angew. Chem., Int. Ed. 2005, 44, 3867-3870. 


\section{Synthetic Procedures}

(R)-trans-Pt(II)-PPh ${ }_{3}$ Diamidine $(\boldsymbol{R})$-3a. A catalytic amount of $\mathrm{CuI}(9.5 \mathrm{mg}, 0.05$ mmol) was added to a degassed solution of the monosilylamidine $(R)-5$ (240 mg, 0.40 $\mathrm{mmol})$ and $\mathrm{PtCl}_{2}\left(\mathrm{PPh}_{3}\right)_{2}(158 \mathrm{mg}, 0.20 \mathrm{mmol})$ in $\mathrm{Et}_{2} \mathrm{NH}(40.0 \mathrm{~mL})$. The mixture was stirred under reflux for $4 \mathrm{~h}$. The solvent was removed in vacuo. The residue was dissolved in EtOAc $(50 \mathrm{~mL})$, and the solution was washed in order with $0.5 \mathrm{M} \mathrm{HCl}(2 \mathrm{x}$ $50 \mathrm{~mL})$, water $(50 \mathrm{~mL}), 0.5 \mathrm{M} \mathrm{NaOH}(50 \mathrm{~mL})$, water $(50 \mathrm{~mL})$, and brine $(50 \mathrm{~mL})$. The organic layer was dried over anhydrous $\mathrm{MgSO}_{4}$ then evaporated to dryness. The residue was washed with $n$-hexane $(15 \mathrm{~mL})$ and dried in vacuo to afford the $(R)$-trans$\mathrm{Pt}(\mathrm{II})-\mathrm{PPh}_{3}$ diamidine $(R)-3 \mathbf{a}\left(305 \mathrm{mg}, 79 \%\right.$ yield) as a pale yellow solid. ${ }^{1} \mathrm{H} \mathrm{NMR}$ $\left(500 \mathrm{MHz}, \mathrm{CDCl}_{3},(R)-3 \mathrm{a}(6.2 \mathrm{mM}), \mathrm{CH}_{3} \mathrm{CO}_{2} \mathrm{H}(56 \mathrm{mM}), 25{ }^{\circ} \mathrm{C}\right.$ ) (for the spectrum in the absence of acetic acid, see Figure S1a): $\delta 12.83$ (br d, 4H, N-H), 7.81-7.73 (m, 13H, ArH), 7.65 (t, $J=7.8 \mathrm{~Hz}, 2 \mathrm{H}, \mathrm{ArH}), 7.48-7.43$ (m, 6H, ArH), 7.41-7.34 (m, 17H, ArH), 7.25-7.11 (m, 14H, ArH), 7.02 (m, 8H, ArH), 6.64 (d, $J=8.5 \mathrm{~Hz}, 4 \mathrm{H}, \mathrm{ArH}), 6.28$ (d, $J=$

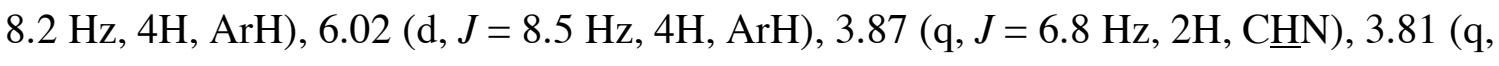
$J=6.7 \mathrm{~Hz}, 2 \mathrm{H}, \underline{\mathrm{H}} \underline{\mathrm{H}}), 2.10\left(\mathrm{~s}, 27 \mathrm{H}, \mathrm{CH}_{3} \mathrm{CO}_{2}\right), 0.68\left(\mathrm{~d}, J=6.9 \mathrm{~Hz}, 6 \mathrm{H}, \underline{\mathrm{CH}}_{\underline{3}} \mathrm{CHN}\right), 0.56$ $\left(\mathrm{d}, J=6.6 \mathrm{~Hz}, 6 \mathrm{H}, \underline{\mathrm{CH}}_{3} \mathrm{CHN}\right), 0.25$ (s, $\left.18 \mathrm{H}, \mathrm{TMS}\right) .{ }^{13} \mathrm{C} \mathrm{NMR}\left(125 \mathrm{MHz}, \mathrm{CDCl}_{3},(R)-\right.$ 3a $\left.(6.2 \mathrm{mM}), \mathrm{CH}_{3} \mathrm{CO}_{2} \mathrm{H}(56 \mathrm{mM}), 25{ }^{\circ} \mathrm{C}\right): \delta 176.82,162.81,142.54,142.46,142.41$, $141.15,138.25,135.06$ (vt, $J=6.0 \mathrm{~Hz}$ ), 133.89, 132.18, 131.58, 131.26, 131.18, 131.04, $130.80,130.38,130.24,129.73,128.95,128.85,128.58,128.39,127.88$ (vt, $J=5.2 \mathrm{~Hz}$ ), $127.76,127.32$, 126.60, 126.54, 123.15, 122.16, 104.21, 95.89, 55.40, 22.17, 21.98, 21.53, -0.11. ${ }^{31} \mathrm{P}$ NMR (200 MHz, $\mathrm{CDCl}_{3},(R)-3 \mathbf{a}(1.0 \mathrm{mM}), \mathrm{CH}_{3} \mathrm{CO}_{2} \mathrm{H}(39 \mathrm{mM}), 25$ $\left.{ }^{\circ} \mathrm{C}\right): \delta 18.80(J=2627 \mathrm{~Hz}) . \quad \mathrm{FT}-\mathrm{IR}\left(\mathrm{KBr}, \mathrm{cm}^{-1}\right): v=3432(\mathrm{~N}-\mathrm{H}), 2156(\mathrm{C}=\mathrm{C}), 2108$ $(\mathrm{C}=\mathrm{C}), 1636(\mathrm{C}=\mathrm{N}) . \quad \mathrm{MS}(\mathrm{ESI}+): \mathrm{m} / \mathrm{z}=1919.89[\mathrm{M}+\mathrm{H}]^{+}, 960.44[\mathrm{M}+2 \mathrm{H}]^{2+}$. Anal. Calcd for $\mathrm{C}_{120} \mathrm{H}_{108} \mathrm{~N}_{4} \mathrm{P}_{2} \mathrm{PtSi}_{2} \cdot\left(\mathrm{C}_{6} \mathrm{H}_{14}\right)_{1}: \mathrm{C}, 75.46 ; \mathrm{H}, 6.13 ; \mathrm{N}, 2.79$. Found: C, 75.65; H, $5.96 ; \mathrm{N}, 2.69$.

(S)-trans-Pt(II)- $\mathbf{P P h}_{3}$ Diamidine (S)-3a. The title compound was prepared from the monosilyl amidine $(S)-\mathbf{5 a}$ in the same way for $(\boldsymbol{R})$-3a and was obtained in $87 \%$ yield as a pale yellow solid. ${ }^{1} \mathrm{H}$ NMR $\left(500 \mathrm{MHz}, \mathrm{CDCl}_{3},(S)-3 \mathbf{a}(8.5 \mathrm{mM}), \mathrm{CH}_{3} \mathrm{CO}_{2} \mathrm{H}(34 \mathrm{mM})\right.$, $25^{\circ} \mathrm{C}$ ): $\delta 13.08$ (br s, 4H, N-H), 7.81-7.74 (m, 13H, ArH), 7.65 (t, J=7.8 Hz, 2H, ArH), 7.49-7.43 (m, 6H, ArH), 7.42-7.34 (m, 17H, ArH), 7.25-7.11 (m, 14H, ArH), 7.03 (m, 
8H, ArH), 6.64 (d, J=8.2 Hz, 4H, ArH), 6.29 (d, J=8.2 Hz, 4H, ArH), 6.02 (d, J = 8.2

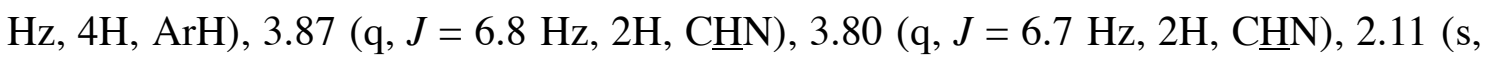
$12 \mathrm{H}, \mathrm{CH}_{3} \mathrm{CO}_{2}$ ), 0.68 (d, $\left.J=6.9 \mathrm{~Hz}, 6 \mathrm{H}, \underline{\mathrm{CH}}_{3} \mathrm{CHN}\right), 0.57$ (d, J=6.9 Hz, 6H, $\left.\underline{\mathrm{CH}}_{2} \mathrm{CHN}\right)$, 0.25 (s, 18H, TMS). ${ }^{13} \mathrm{C}$ NMR (125 MHz, $\mathrm{CDCl}_{3},(S)-3 \mathrm{a}(8.5 \mathrm{mM}), \mathrm{CH}_{3} \mathrm{CO}_{2} \mathrm{H}(34$ $\left.\mathrm{mM}), 25^{\circ} \mathrm{C}\right): \delta 177.29,162.74,142.72,142.64,142.41,141.15,138.31,135.06$ (vt, $J=$ $6.0 \mathrm{~Hz}), 133.95,132.16,131.50,131.25,131.19,131.02,130.79,130.39,130.23$, 129.71, 128.92, 128.82, 128.53, 128.40, 127.88 (vt, $J=5.2 \mathrm{~Hz}), 127.69,127.34,126.61$, $126.54,123.11,122.28,104.24,95.85,55.36,22.90,22.29,22.10,-0.10 . \quad{ }^{31} \mathrm{P}$ NMR $\left(200 \mathrm{MHz}, \mathrm{CDCl}_{3},(S)-\mathbf{3 a}(8.5 \mathrm{mM}), \mathrm{CH}_{3} \mathrm{CO}_{2} \mathrm{H}(34 \mathrm{mM}), 25{ }^{\circ} \mathrm{C}\right): \delta 18.78(\mathrm{~J}=2627 \mathrm{~Hz})$. FT-IR $\left(\mathrm{KBr}, \mathrm{cm}^{-1}\right): v=3432(\mathrm{~N}-\mathrm{H}), 2156(\mathrm{C} \equiv \mathrm{C}), 2108(\mathrm{C} \equiv \mathrm{C}), 1636(\mathrm{C}=\mathrm{N}) . \quad \mathrm{MS}$ $(\mathrm{ESI}+): \mathrm{m} / \mathrm{z}=1920.08[\mathrm{M}+\mathrm{H}]^{+}, \quad 960.55[\mathrm{M}+2 \mathrm{H}]^{2+}$. Anal. Calcd for $\mathrm{C}_{120} \mathrm{H}_{108} \mathrm{~N}_{4} \mathrm{P}_{2} \mathrm{PtSi}_{2} \cdot\left(\mathrm{H}_{2} \mathrm{O}\right)_{1}$ : C, 74.39; H, 5.72; N, 2.89. Found: C, 74.21; H, 5.55; N, 2.64.

trans-Pt(II)-PPh ${ }_{3}$ Dicarboxylic Acid 4a. A catalytic amount of CuI (12 mg, 0.06 mmol) was added to a degassed solution of the monosilyl carboxylic acid 6 (197 $\mathrm{mg}$, $0.50 \mathrm{mmol})$ and $\mathrm{PtCl}_{2}\left(\mathrm{PPh}_{3}\right)_{2}(198 \mathrm{mg}, 0.25 \mathrm{mmol})$ in $\mathrm{Et}_{2} \mathrm{NH}(50.0 \mathrm{~mL})$. The mixture was stirred under reflux for $3 \mathrm{~h}$. The solvent was removed in vacuo. The residue was dissolved in EtOAc $(50 \mathrm{~mL})$ and $0.5 \mathrm{M} \mathrm{HCl}(50 \mathrm{~mL})$, and the aqueous layer was extracted with EtOAc $(40 \mathrm{~mL})$. The mixed organic layer was washed with $0.5 \mathrm{M} \mathrm{HCl}$ $(2 \times 40 \mathrm{~mL})$, water $(40 \mathrm{~mL})$, and brine $(40 \mathrm{~mL})$. The organic layer was dried over anhydrous $\mathrm{MgSO}_{4}$ and evaporated to dryness. The residue was washed with $n$-hexane $(15 \mathrm{~mL})$ and dried in vacuo to afford the trans-Pt(II)- $\mathrm{PPh}_{3}$ dicarboxylic acid $\mathbf{4 a}(343 \mathrm{mg}$, $91 \%$ yield) as a pale yellow solid. ${ }^{1} \mathrm{H}$ NMR (500 MHz, DMSO- $d_{6}, 4 \mathbf{a}$ (9.6 mM), 25 $\left.{ }^{\circ} \mathrm{C}\right): \delta 12.83$ (br s, 2H, O-H), 7.78-7.72 (m, 12H, ArH), 7.53-7.46 (m, 25H, ArH), 7.36 $(\mathrm{d}, J=8.5 \mathrm{~Hz}, 3 \mathrm{H}, \mathrm{ArH}), 7.30(\mathrm{~d}, J=7.7 \mathrm{~Hz}, 2 \mathrm{H}, \operatorname{ArH}), 7.25(\mathrm{~d}, J=7.1 \mathrm{~Hz}, 2 \mathrm{H}, \operatorname{ArH})$, $6.97(\mathrm{~d}, J=8.2 \mathrm{~Hz}, 4 \mathrm{H}, \mathrm{ArH}), 6.18$ (d, $J=8.2 \mathrm{~Hz}, 4 \mathrm{H}, \mathrm{ArH}), 0.23$ (s, 18H, TMS). ${ }^{13} \mathrm{C}$ NMR (125 MHz, DMSO- $\left.d_{6}, 4 a(9.6 \mathrm{mM}), 25{ }^{\circ} \mathrm{C}\right): \delta 169.93,140.77,138.59,137.88$, $136.48,134.42$ (vt, $J=6.4 \mathrm{~Hz}), 133.34,131.50,131.10,130.87,130.67,130.05,129.06$, $128.97,128.60,128.40,128.19$ (vt, $J=5.2 \mathrm{~Hz}$ ), 127.38, 127.32, 121.30, 112.03, 104.88, 94.92, -0.11. ${ }^{31} \mathrm{P}$ NMR (200 MHz, $\left.\mathrm{CDCl}_{3}, 4 \mathbf{a}(1.0 \mathrm{mM}), 25{ }^{\circ} \mathrm{C}\right): \delta 18.84(\mathrm{~J}=2641 \mathrm{~Hz})$. FT-IR $\left(\mathrm{KBr}, \mathrm{cm}^{-1}\right): v=3448(\mathrm{O}-\mathrm{H}), 2155(\mathrm{C}=\mathrm{C}), 2107(\mathrm{C}=\mathrm{C}), 1728(\mathrm{C}=\mathrm{O}), 1704$ $(\mathrm{C}=\mathrm{O})$. MS (ESI-): $\mathrm{m} / \mathrm{z}=1505.63[\mathrm{M}-\mathrm{H}]^{-}, 752.32[\mathrm{M}-2 \mathrm{H}]^{2-}$. Anal. Calcd for $\mathrm{C}_{88} \mathrm{H}_{72} \mathrm{O}_{4} \mathrm{P}_{2} \mathrm{PtSi}_{2} \cdot\left(\mathrm{H}_{2} \mathrm{O}\right)_{2}: \mathrm{C}, 68.51 ; \mathrm{H}, 4.97$. Found: $\mathrm{C}, 68.60 ; \mathrm{H}, 4.74$. 
(R)-trans-Pt(II)-PPh ${ }_{3}$ duplex $(\boldsymbol{R})-3 \mathbf{a} \cdot 4 \mathbf{a} . \quad$ The $(R)$-trans-Pt(II)- $\mathrm{PPh}_{3}$ diamidine $(R)-3 \mathbf{a}$ $(19.2 \mathrm{mg}, 0.0100 \mathrm{mmol})$ and the trans-Pt(II)- $\mathrm{PPh}_{3}$ carboxylic acid $4 \mathbf{a}(15.1 \mathrm{mg}, 0.0100$ mmol) were dissolved in $\mathrm{CHCl}_{3}(1.0 \mathrm{~mL})$. The solution was filtered through a membrane filter $(0.45 \mu \mathrm{m})$. The filtrate was evaporated to dryness. The residue was washed with $\mathrm{MeOH}(2 \times 0.5 \mathrm{~mL})$ and dried in vacuo to afford the trans-Pt(II)- $\mathrm{PPh}_{3}$ duplex $(R)-3 \mathbf{3 a} \cdot 4 \mathbf{a}\left(31 \mathrm{mg}, 91 \%\right.$ yield) as a yellow solid. ${ }^{1} \mathrm{H} \mathrm{NMR}\left(500 \mathrm{MHz}, \mathrm{CDCl}_{3}\right.$, $(R)-3 a \cdot 4 a(1.7 \mathrm{mM}), 25{ }^{\circ} \mathrm{C}$ ) (see Figure $\mathrm{S} 1 \mathrm{~b}$ ): Most of the signals were highly broadened, suggesting that the molecular motion of the complexes is highly restricted probably due to the steric repulsion of triphenylphosphine ligands; ${ }^{31} \mathrm{P}$ NMR $(200 \mathrm{MHz}$, $\left.\mathrm{CDCl}_{3},(R)-\mathbf{3 a} \cdot 4 \mathbf{a}(1.7 \mathrm{mM}), 25{ }^{\circ} \mathrm{C}\right): \delta 19.28(\mathrm{~J}=2650 \mathrm{~Hz}), 18.52(\mathrm{~J}=2662 \mathrm{~Hz})$; FT-IR $\left(\mathrm{KBr}, \mathrm{cm}^{-1}\right): v=3421(\mathrm{~N}-\mathrm{H}), 2155(\mathrm{C}=\mathrm{C}), 2106(\mathrm{C}=\mathrm{C}), 1636 . \quad$ MS (ESI+) (see Figure $\mathrm{S} 2): \mathrm{m} / \mathrm{z}=3426.51[\mathrm{M}+\mathrm{H}]^{+}, \quad 1713.77 \quad[\mathrm{M}+2 \mathrm{H}]^{2+}$. Anal. Calcd for $\mathrm{C}_{208} \mathrm{H}_{180} \mathrm{~N}_{4} \mathrm{O}_{4} \mathrm{P}_{4} \mathrm{Pt}_{2} \mathrm{Si}_{4} \cdot\left(\mathrm{CHCl}_{3}\right)_{1}: \mathrm{C}, 70.80 ; \mathrm{H}, 5.15 ; \mathrm{N}, 1.58$. Found: C, 70.61; H, 5.14; N, 1.49 .

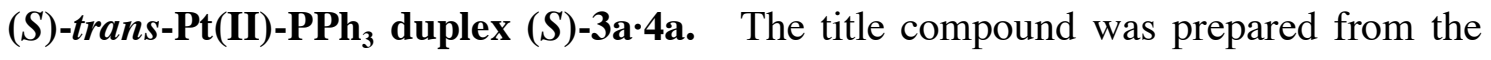
(S)-trans-Pt(II)- $\mathrm{PPh}_{3}$ diamidine $(S)-3 \mathbf{a}(19.2 \mathrm{mg}, 0.01 \mathrm{mmol})$ and the trans-Pt(II)- $\mathrm{PPh}_{3}$ carboxylic acid $\mathbf{4 a}(15.1 \mathrm{mg}, 0.01 \mathrm{mmol})$ in the same way for $(R)-\mathbf{3 a} \cdot \mathbf{4 a}$ and obtained in $82 \%$ yield as a yellow solid. ${ }^{31} \mathrm{P} \mathrm{NMR}\left(200 \mathrm{MHz}, \mathrm{CDCl}_{3},(S)-\mathbf{3 a} \cdot \mathbf{4 a}(2.9 \mathrm{mM}), 25{ }^{\circ} \mathrm{C}\right)$ : $\delta 19.23(J=2672 \mathrm{~Hz}), 18.51(J=2674 \mathrm{~Hz})$; FT-IR $\left(\mathrm{KBr}, \mathrm{cm}^{-1}\right): v=3434(\mathrm{~N}-\mathrm{H}), 2155$ $(\mathrm{C} \equiv \mathrm{C}), 2106(\mathrm{C} \equiv \mathrm{C}), 1636 . \quad \mathrm{MS}(\mathrm{ESI}+): \mathrm{m} / \mathrm{z}=3426.55[\mathrm{M}+\mathrm{H}]^{+}, 1713.94[\mathrm{M}+2 \mathrm{H}]^{2+}$. Anal. Calcd for $\mathrm{C}_{208} \mathrm{H}_{180} \mathrm{~N}_{4} \mathrm{O}_{4} \mathrm{P}_{4} \mathrm{Pt}_{2} \mathrm{Si}_{4} \cdot\left(\mathrm{CHCl}_{3}\right)_{1.8}: \mathrm{C}, 69.21 ; \mathrm{H}, 5.03 ; \mathrm{N}, 1.54$. Found: $\mathrm{C}$, $69.13 ; \mathrm{H}, 5.10 ; \mathrm{N}, 1.45$.

$(\boldsymbol{R})$-cis-Pt(II)-dppee Diamidine $\quad(\boldsymbol{R})$-3b. $\quad$ A mixture of the cis-1,2bis(diphenylphosphinoethylene) (dppee) $(28 \mathrm{mg}, 0.07 \mathrm{mmol})$ and the (R)-cis-Pt(II)-PPh diamidine $(R)-3 \mathbf{b}(134 \mathrm{mg}, 0.07 \mathrm{mmol})$ in $\mathrm{CH}_{2} \mathrm{Cl}_{2}(7.0 \mathrm{~mL})$ was stirred at ambient temperature for $17 \mathrm{~h}$. The solvent was removed in vacuo. The residue was then washed with $n$-hexane $(7 \mathrm{~mL})$ and dried in vacuo to afford the $(R)$-cis-Pt(II)-dppee diamidine $(R)-3 \mathbf{b}\left(124 \mathrm{mg}, 99 \%\right.$ yield) as a pale yellow solid. ${ }^{1} \mathrm{H}$ NMR (500 MHz, $\mathrm{CDCl}_{3},(R)-3 \mathbf{b}(1.0 \mathrm{mM}), \mathrm{CH}_{3} \mathrm{CO}_{2} \mathrm{H}(41 \mathrm{mM}), 25^{\circ} \mathrm{C}$ ) (for the spectrum in the absence of acetic acid, see Figure S3a): $\delta 12.77$ (br s, 4H, N-H), 7.83-7.79 (m, 4H, ArH), 
7.74-7.67 (m, 6H, ArH), 7.58-7.36 (m, 21H, ArH), 7.25-7.13 (m, 13H, ArH), 7.02 (m, $8 \mathrm{H}, \mathrm{ArH}), 6.92(\mathrm{~d}, J=8.5 \mathrm{~Hz}, 4 \mathrm{H}, \mathrm{ArH}), 6.65$ (d, $J=8.2 \mathrm{~Hz}, 4 \mathrm{H}, \mathrm{ArH}), 6.47$ (d, $J=8.5$ $\mathrm{Hz}, 4 \mathrm{H}, \mathrm{ArH}), 3.90(\mathrm{~m}, J=6.5 \mathrm{~Hz}, 4 \mathrm{H}, \mathrm{CHN}), 2.10$ (s, $\left.124 \mathrm{H}, \mathrm{CH}_{3} \mathrm{CO}_{2}\right), 0.70$ (d, J = 6.9 $\mathrm{Hz}, 6 \mathrm{H}, \underline{\mathrm{CH}}_{3} \mathrm{CHN}$ ), 0.58 (d, $\left.J=6.6 \mathrm{~Hz}, 6 \mathrm{H}, \mathrm{CH}_{3} \mathrm{CHN}\right), 0.25$ (s, 18H, TMS). ${ }^{13} \mathrm{C} \mathrm{NMR}$ $\left(125 \mathrm{MHz}, \mathrm{CDCl}_{3},(R)-3 \mathbf{b}(5.1 \mathrm{mM}), \mathrm{CH}_{3} \mathrm{CO}_{2} \mathrm{H}(50 \mathrm{mM}), 25{ }^{\circ} \mathrm{C}\right): \delta 176.62,162.85$, 147.13-146.14 (m), 142.55, 142.40, 141.19, 138.32, 134.46, 133.71, 133.62, 133.16, 133.07, 132.16, 132.09, 131.66, 131.48, 131.42, 130.45, 129.84, 129.62, 129.38, 129.16, 128.98, 128.94, 128.90, 128.89, 128.79, 128.57, 128.47, 128.39, 127.82, 127.77, 126.61, $126.53,123.11,122.22,104.26,95.82,55.44,22.20,21.85,21.61,-0.10 . \quad{ }^{31} \mathrm{P}$ NMR $\left(200 \mathrm{MHz}, \mathrm{CDCl}_{3},(R)-\mathbf{3 b}(1.0 \mathrm{mM}), \mathrm{CH}_{3} \mathrm{CO}_{2} \mathrm{H}(20.7 \mathrm{mM}), 25{ }^{\circ} \mathrm{C}\right): \delta 52.71(\mathrm{~J}=2277$ Hz). FT-IR $\left(\mathrm{KBr}, \mathrm{cm}^{-1}\right): v=3430(\mathrm{~N}-\mathrm{H}), 2155(\mathrm{C} \equiv \mathrm{C}), 2114(\mathrm{C} \equiv \mathrm{C}), 1636(\mathrm{C}=\mathrm{N})$. MS (ESI+): $\mathrm{m} / \mathrm{z}=1791.80[\mathrm{M}+\mathrm{H}]^{+}, 896.40[\mathrm{M}+2 \mathrm{H}]^{2+}$. Anal. Calcd for $\mathrm{C}_{110} \mathrm{H}_{100} \mathrm{~N}_{4}$ $\mathrm{P}_{2} \mathrm{PtSi}_{2} \cdot\left(\mathrm{H}_{2} \mathrm{O}\right)_{1}: \mathrm{C}, 73.03 ; \mathrm{H}, 5.68 ; \mathrm{N}, 3.10$. Found: C, 73.12; H, 5.48; N, 2.82.

(S)-cis-Pt(II)-dppee Diamidine (S)-3b. The title compound was prepared from dppee and the $(S)$-trans-Pt(II)- $\mathrm{PPh}_{3}$ diamidine $(S)-\mathbf{3 a}$ in the same way for $(R)-\mathbf{3 b}$ and obtained in $91 \%$ yield as a pale yellow solid. ${ }^{1} \mathrm{H}$ NMR (500 $\mathrm{MHz}, \mathrm{CDCl}_{3},(S)-3 \mathbf{b}(4.6 \mathrm{mM})$, $\left.\mathrm{CH}_{3} \mathrm{CO}_{2} \mathrm{H}(129 \mathrm{mM}), 25{ }^{\circ} \mathrm{C}\right): \delta 12.52$ (br s, 4H, N-H), 7.86-7.78 (m, 4H, ArH), 7.74-7.66 (m, 6H, ArH), 7.59-7.36 (m, 21H, ArH), 7.26-7.12 (m, 13H, ArH), 7.01 (m, $8 \mathrm{H}, \mathrm{ArH}), 6.92(\mathrm{~d}, J=8.2 \mathrm{~Hz}, 4 \mathrm{H}, \mathrm{ArH}), 6.64(\mathrm{~d}, J=8.2 \mathrm{~Hz}, 4 \mathrm{H}, \mathrm{ArH}), 6.46$ (d, $J=8.2$ $\mathrm{Hz}, 4 \mathrm{H}, \mathrm{ArH}), 3.90$ (m, 4H, CㅌN), 2.09 (s, 84H, $\mathrm{CH}_{3} \mathrm{CO}_{2}$ ), 0.70 (d, J = $6.9 \mathrm{~Hz}, 6 \mathrm{H}$, $\left.\underline{\mathrm{CH}}_{2} \mathrm{CHN}\right), 0.58$ (d, $\left.J=6.6 \mathrm{~Hz}, 6 \mathrm{H}, \underline{\mathrm{CH}}_{3} \mathrm{CHN}\right), 0.25$ (s, 18H, TMS). ${ }^{13} \mathrm{C} \mathrm{NMR}(125$ $\left.\mathrm{MHz}, \mathrm{CDCl}_{3},(S)-3 \mathbf{b}(4.6 \mathrm{mM}), \mathrm{CH}_{3} \mathrm{CO}_{2} \mathrm{H}(129 \mathrm{mM}), 25{ }^{\circ} \mathrm{C}\right): \delta 176.97,162.93$, 147.07-146.01 (m), 142.39, 141.20, 138.26, 134.43, 133.70, 133.60, 133.17, 133.08, $132.17,132.10,131.73,131.49,131.43,130.46,129.86,129.61,129.37,129.14,129.06$, $128.96,128.93,128.89,128.79,128.59,128.49,128.38,127.87,127.77,126.61,126.52$, 123.16, 122.12, 104.23, 95.86, 55.48, 22.11, 21.77, 21.05, $-0.11 . \quad{ }^{31} \mathrm{P}$ NMR (200 MHz, $\left.\mathrm{CDCl}_{3},(S)-3 \mathbf{b}(4.6 \mathrm{mM}), \mathrm{CH}_{3} \mathrm{CO}_{2} \mathrm{H}(129 \mathrm{mM}), 25^{\circ} \mathrm{C}\right): \delta 52.72(\mathrm{~J}=2277 \mathrm{~Hz}) . \quad$ FT-IR $\left(\mathrm{KBr}, \mathrm{cm}^{-1}\right): v=3432(\mathrm{~N}-\mathrm{H}), 2155(\mathrm{C} \equiv \mathrm{C}), 2114(\mathrm{C} \equiv \mathrm{C}), 1636(\mathrm{C}=\mathrm{N}) . \quad \mathrm{MS}(\mathrm{ESI}+): \mathrm{m} / \mathrm{z}$ $=1791.98[\mathrm{M}+\mathrm{H}]^{+}, 896.50[\mathrm{M}+2 \mathrm{H}]^{2+}$. Anal. Calcd for $\mathrm{C}_{110} \mathrm{H}_{100} \mathrm{~N}_{4} \mathrm{P}_{2} \mathrm{PtSi}_{2} \cdot\left(\mathrm{H}_{2} \mathrm{O}\right)_{2}: \mathrm{C}$, 72.31; H, 5.74; N, 3.07. Found: C, 72.37; H, 5.50; N, 2.89.

cis-Pt(II)-dppee Dicarboxylic Acid 4b. A mixture of dppee (40 mg, $0.10 \mathrm{mmol}$ ) and 
the trans-Pt(II)- $\mathrm{PPh}_{3}$ dicarboxylic acid $3 \mathbf{b}(151 \mathrm{mg}, 0.10 \mathrm{mmol})$ in $\mathrm{CH}_{2} \mathrm{Cl}_{2}(10.0 \mathrm{~mL})$ was stirred at ambient temperature for $30 \mathrm{~min}$. The solvent was removed in vacuo. The residue was then washed with $n$-hexane $(12 \mathrm{~mL})$ and dried in vacuo to afford the cis-Pt(II)-dppee dicarboxylic acid $\mathbf{4 b}$ (138 $\mathrm{mg}$, quantitative yield) as a yellow solid. ${ }^{1} \mathrm{H}$ NMR (500 MHz, $\left.\mathrm{CDCl}_{3}, \mathbf{4 b}(6.5 \mathrm{mM}), 25{ }^{\circ} \mathrm{C}\right): \delta 7.87-7.79(\mathrm{~m}, 8 \mathrm{H}, \mathrm{ArH}), 7.50-7.02$ (m, 36H, ArH), 0.26 (s, 18H, TMS). ${ }^{13} \mathrm{C} \mathrm{NMR} \mathrm{(125} \mathrm{MHz,} \mathrm{CDCl}_{3}, 4 \mathbf{b}(6.5 \mathrm{mM}), 25$ $\left.{ }^{\circ} \mathrm{C}\right): \delta 172.01,147.18-146.14(\mathrm{~m}), 140.78,140.17(\mathrm{~m}), 139.41(\mathrm{~m}), 137.16(\mathrm{~m}), 133.81$, 133.67, 133.45, 133.36, 132.14, 132.06, 132.00, 131.99, 131.84, 131.36, 131.22, 130.50, 130.03, 129.94, 129.57, 129.17, 129.14, 128.90, 128.81, 128.55, 128.47, 127.82, 127.50, 122.26, 105.15, 94.74, -0.02. ${ }^{31} \mathrm{P}$ NMR (200 MHz, $\left.\mathrm{CDCl}_{3}, 4 \mathbf{b}(1.7 \mathrm{mM}), 25{ }^{\circ} \mathrm{C}\right): \delta$ $52.60(\mathrm{~J}=2300 \mathrm{~Hz})$. FT-IR $\left(\mathrm{KBr}, \mathrm{cm}^{-1}\right): v=3435(\mathrm{O}-\mathrm{H}), 2155(\mathrm{C} \equiv \mathrm{C}), 2113(\mathrm{C} \equiv \mathrm{C})$, $1725(\mathrm{C}=\mathrm{O}) . \quad \mathrm{m} / \mathrm{z}=1377.61[\mathrm{M}-\mathrm{H}]^{-}, 688.28[\mathrm{M}-2 \mathrm{H}]^{2-}$. Anal. Calcd for $\mathrm{C}_{78} \mathrm{H}_{64} \mathrm{O}_{4} \mathrm{P}_{2} \mathrm{PtSi}_{2} \cdot\left(\mathrm{H}_{2} \mathrm{O}\right)_{1}: \mathrm{C}, 67.08 ; \mathrm{H}, 4.76$. Found: $\mathrm{C}, 67.05 ; \mathrm{H}, 4.66$.

(R)-cis-Pt(II)-dppee Duplex $(\boldsymbol{R})$-3b·4b. The $(R)$-cis-Pt(II)-dppee diamidine $(R)$-3b $(17.9 \mathrm{mg}, 0.01 \mathrm{mmol})$ and the $c i s-\mathrm{Pt}(\mathrm{II})$-dppee dicarboxylic acid $4 \mathbf{b}$ (13.8 $\mathrm{mg}, 0.01$ mmol) were dissolved in $\mathrm{CHCl}_{3}(1.0 \mathrm{~mL})$. The solution was filtered through a membrane filter $(0.45 \mu \mathrm{m})$. The filtrate was evaporated to dryness. The residue was then washed with $\mathrm{MeOH}(2 \times 0.5 \mathrm{~mL})$ and dried in vacuo to afford the duplex $(R)-\mathbf{3 b} \cdot \mathbf{4 b}$ (25 mg, 78\% yield) as a pale yellow solid. ${ }^{1} \mathrm{H} \mathrm{NMR}\left(500 \mathrm{MHz}, \mathrm{CDCl}_{3},(R)-\mathbf{3 b} \cdot \mathbf{4 b}(1.0\right.$ $\mathrm{mM}$ ), $25^{\circ} \mathrm{C}$ ) (see Figure S3b): $\delta 13.26($ br d, 2H, N-H), 12.87 (br d, 2H, N-H), 8.10-6.06 (m, 108H, ArH), 3.74 (m, 2H, C $\underline{H N}), 3.56$ (m, 2H, CHN), 0.61 (d, J = 6.0 Hz, $6 \mathrm{H}, \mathrm{CH}_{3} \mathrm{CHN}$ ), 0.38 (d, J = 6.3 Hz, 6H, $\underline{\mathrm{CH}}_{3} \mathrm{CHN}$ ), 0.29 (s, 18H, TMS), 0.23 (s, 18H, TMS). $\left.\quad{ }^{13} \mathrm{C} \mathrm{NMR} \mathrm{(125} \mathrm{MHz,} \mathrm{CDCl}_{3},(R)-3 \mathbf{b} \cdot 4 \mathbf{b}(5.9 \mathrm{mM}), 25{ }^{\circ} \mathrm{C}\right): \delta 176.88,162.76$, 147.16-146.36 (m), 143.65, 142.78, 142.22, 141.56, 141.07, 139.96, 139.05, 138.61, 138.36, 137.54, 134.24-125.66 (m), 122.88, 122.69, 121.15, 111.77, 111.51, 106.17, 104.40, 95.70, 94.28, 55.34, 55.20, 21.66, 21.58, 0.16, -0.06. ${ }^{31} \mathrm{P}$ NMR (200 MHz, $\left.\mathrm{CDCl}_{3},(R)-\mathbf{3 b} \cdot \mathbf{4 b} \quad(1.0 \mathrm{mM}), 25{ }^{\circ} \mathrm{C}\right): \delta 52.92(J=2264 \mathrm{~Hz}), 51.92(J=2258 \mathrm{~Hz})$. FT-IR $\left(\mathrm{KBr}, \mathrm{cm}^{-1}\right): v=3431(\mathrm{~N}-\mathrm{H}), 2155(\mathrm{C} \equiv \mathrm{C}), 2113(\mathrm{C} \equiv \mathrm{C}), 1634$. MS (ESI+) (see Figure S4): $\mathrm{m} / \mathrm{z}=3170.39[\mathrm{M}+\mathrm{H}]^{+}, 1585.66[\mathrm{M}+2 \mathrm{H}]^{2+}$. Anal. Calcd for $\mathrm{C}_{188} \mathrm{H}_{164} \mathrm{~N}_{4} \mathrm{O}_{4} \mathrm{P}_{4} \mathrm{Pt}_{2} \mathrm{Si}_{4} \cdot\left(\mathrm{H}_{2} \mathrm{O}\right)_{6}: \mathrm{C}, 68.89 ; \mathrm{H}, 5.41 ; \mathrm{N}, 1.71$. Found: C, 68.90; H, 5.16; N, 1.51 . 
(S)-cis-Pt(II)-dppee Duplex $(S)-3 b \cdot 4 b$. The title compound was prepared from the $(S)$-cis-Pt(II)-dppee diamidine $(S)-3 \mathbf{b}(17.9 \mathrm{mg}, 0.01 \mathrm{mmol})$ and the cis-Pt(II)-dppee dicarboxylic acid $\mathbf{4 b}(13.8 \mathrm{mg}, 0.01 \mathrm{mmol})$ in the same way for $(R)-\mathbf{3 b} \cdot \mathbf{4 b}$ and obtained in $98 \%$ yield as a pale yellow solid. ${ }^{1} \mathrm{H} \mathrm{NMR}\left(500 \mathrm{MHz}, \mathrm{CDCl}_{3},(S)-\mathbf{3 b} \cdot \mathbf{4 b}(2.5 \mathrm{mM})\right.$, $25{ }^{\circ} \mathrm{C}$ ): $\delta 13.25$ (br d, 2H, N-H), 12.85 (br d, 2H, N-H), 8.05-6.10 (m, 108H, ArH), 3.74 (m, 2H, CHN), 3.56 (m, 2H, CㅂN), 0.61 (d, $\left.J=6.0 \mathrm{~Hz}, 6 \mathrm{H}, \underline{\mathrm{CH}}_{3} \mathrm{CHN}\right), 0.37$ (d, $J$ $\left.=6.0 \mathrm{~Hz}, 6 \mathrm{H}, \underline{\mathrm{CH}}_{3} \mathrm{CHN}\right), 0.29$ (s, 18H, TMS), 0.23 (s, 18H, TMS). ${ }^{13} \mathrm{C} \mathrm{NMR}(125$ $\left.\mathrm{MHz}, \mathrm{CDCl}_{3},(S)-3 \mathbf{b} \cdot \mathbf{4 b}(5.4 \mathrm{mM}), 25{ }^{\circ} \mathrm{C}\right): \delta$ 176.89, 162.76, 147.18-146.41 (m), $143.62,142.78,142.22,141.56,141.08,139.95,139.05,138.61,138.35,137.53$, 134.35-125.71 (m), 122.88, 122.69, 121.16, 111.77, 111.51, 106.16, 104.40, 95.71, 94.29, 55.34, 55.21, 21.66, 21.60, 0.17, -0.05. ${ }^{31} \mathrm{P} \mathrm{NMR} \mathrm{(200} \mathrm{MHz,} \mathrm{CDCl}_{3},(S)-3 \mathbf{b} \cdot \mathbf{4 b}$ $\left.(1.0 \mathrm{mM}), 25{ }^{\circ} \mathrm{C}\right): \delta 52.92(\mathrm{~J}=2262 \mathrm{~Hz}), 51.93(\mathrm{~J}=2257 \mathrm{~Hz}) . \quad$ FT-IR $\left(\mathrm{KBr}, \mathrm{cm}^{-1}\right): v=$ $3648(\mathrm{~N}-\mathrm{H}), 2155(\mathrm{C} \equiv \mathrm{C}), 2113(\mathrm{C} \equiv \mathrm{C}), 1634,1583 . \quad \mathrm{MS}(\mathrm{ESI}+): \mathrm{m} / \mathrm{z}=3170.35$ $[\mathrm{M}+\mathrm{H}]^{+}, 1585.79[\mathrm{M}+2 \mathrm{H}]^{2+}$. Anal. Calcd for $\mathrm{C}_{188} \mathrm{H}_{164} \mathrm{~N}_{4} \mathrm{O}_{4} \mathrm{P}_{4} \mathrm{Pt}_{2} \mathrm{Si}_{4} \cdot\left(\mathrm{CHCl}_{3}\right)_{1.2}: \mathrm{C}$, 68.59; H, 5.03; N, 1.69. Found: C, 68.61; H, 5.11; N, 1.58. 


\section{Characterization of the trans-Pt(II)-PPh ${ }_{3}$ Duplex 3a.4a}

${ }^{1} \mathrm{H}$ NMR (500 MHz, $\left.\mathrm{CDCl}_{3}, 25{ }^{\circ} \mathrm{C}\right)$ Spectra of $(R)-3 \mathrm{a}$ and $(R)-3 \mathrm{a} \cdot 4 \mathrm{a}$

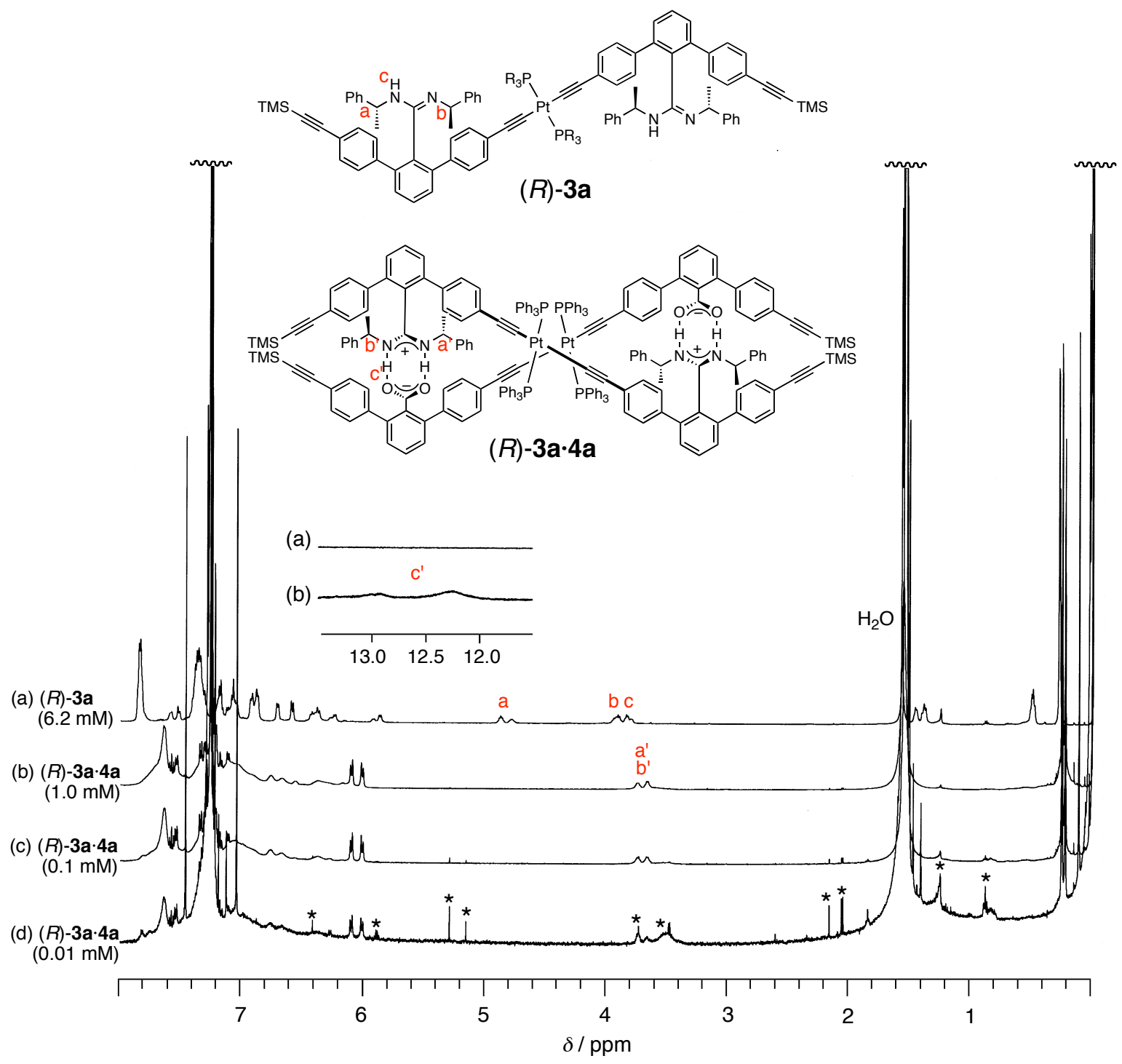

Figure S1. $\quad{ }^{1} \mathrm{H}$ NMR $\left(500 \mathrm{MHz}, \mathrm{CDCl}_{3}, 25{ }^{\circ} \mathrm{C}\right)$ spectra of $(R)-\mathbf{3 a}(6.2 \mathrm{mM})$ (a) and $(R)-3 \mathbf{a} \cdot 4 \mathbf{a}(1.0 \mathrm{mM}(\mathrm{b}), 0.1 \mathrm{mM}(\mathrm{c})$, and $0.01 \mathrm{mM}(\mathrm{d}))$. The asterisks denote impurities contained in the solvent $\mathrm{CDCl}_{3}$. 


\section{ESI-MS Spectrum of $(R)-3 a \cdot 4 a$}

a) Experimental ESI-MS Spectrum of $[(R)-3 \mathbf{a} \cdot 4 \mathbf{a}+2 \mathrm{H}]^{2+}$

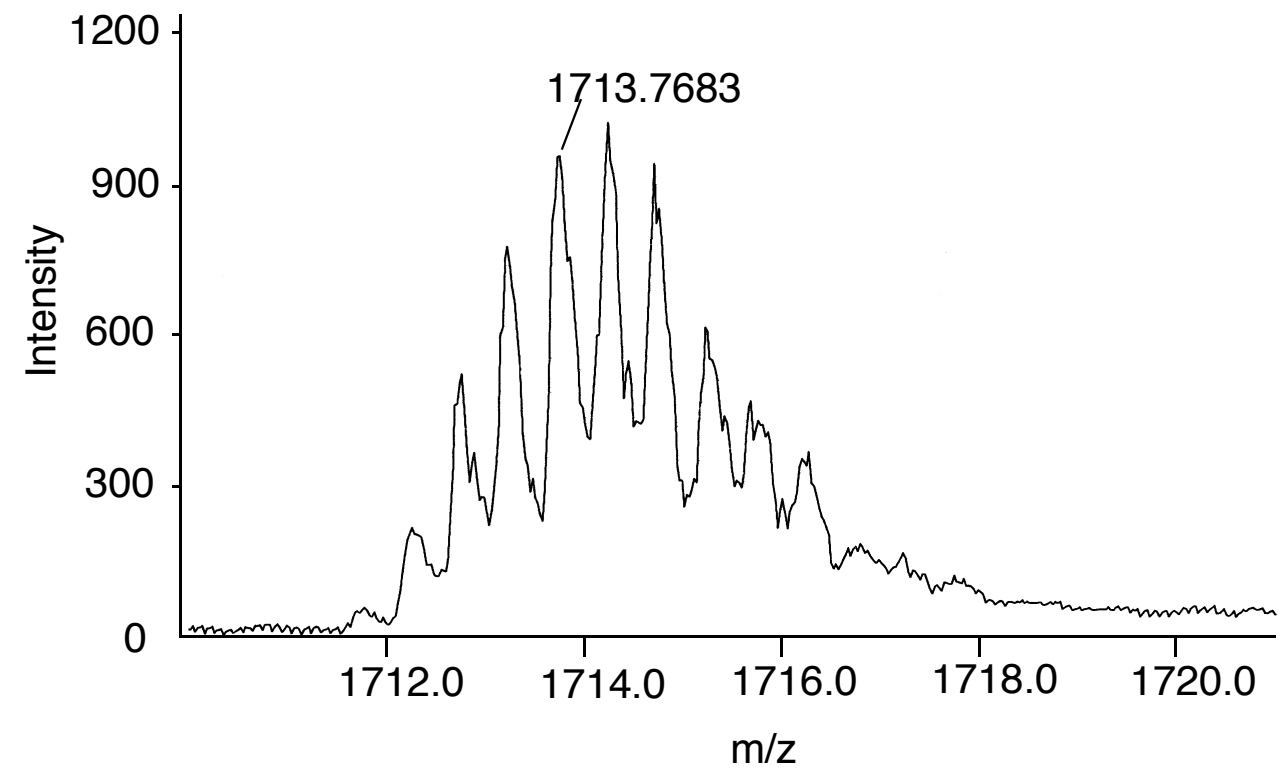

b) Isotope Simulation of $[(R)-3 \mathbf{a} \cdot \mathbf{4 a}+2 \mathrm{H}]^{2+}$

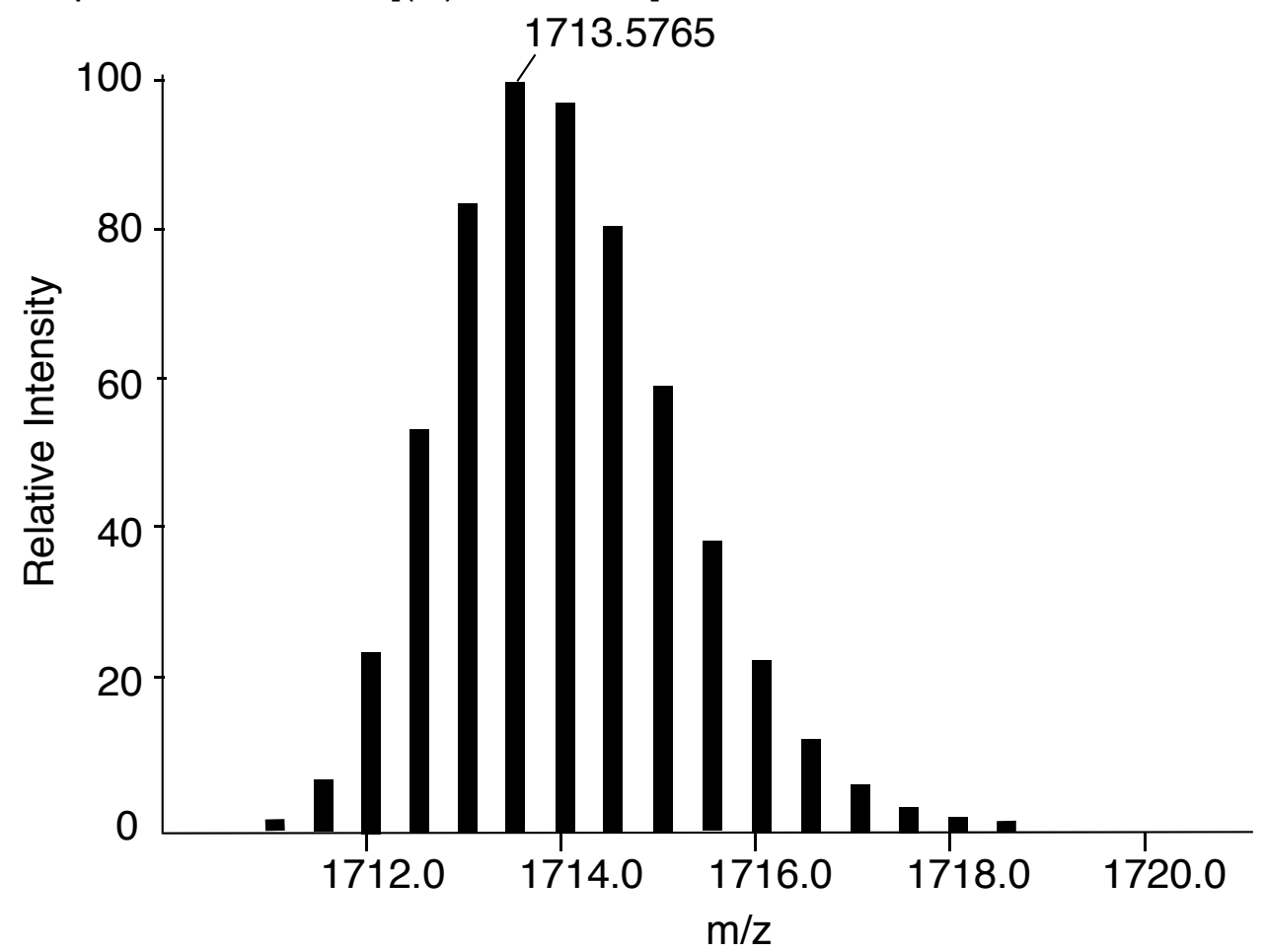

Figure S2. (a) Experimental and (b) simulated ESI mass spectra of $[(R)-\mathbf{3 a} \cdot \mathbf{4 a}+2 \mathrm{H}]^{2+}$. 
5. Characterization of the cis-Pt(II)-dppee Duplex $3 \mathrm{~b} \cdot 4 \mathrm{~b}$

${ }^{1} \mathrm{H}$ NMR (500 MHz, $\left.\mathrm{CDCl}_{3}, 25{ }^{\circ} \mathrm{C}\right)$ Spectra of $(R)-3 \mathrm{~b}$ and $(R)-3 \mathrm{~b} \cdot 4 \mathrm{~b}$
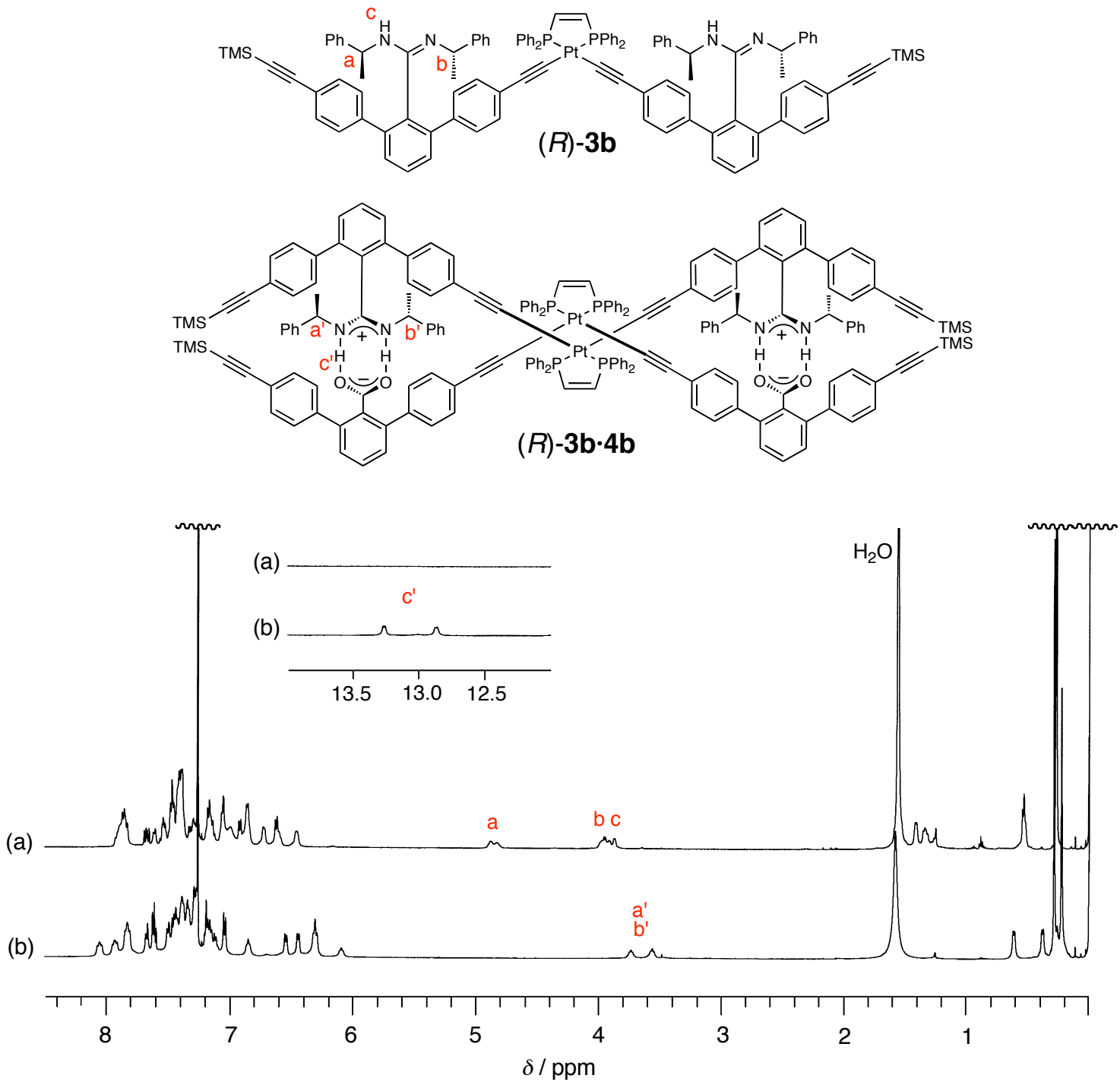

Figure S3. $\quad{ }^{1} \mathrm{H} \mathrm{NMR}\left(500 \mathrm{MHz}, \mathrm{CDCl}_{3}, 25^{\circ} \mathrm{C}\right)$ spectra of (a) $(R)-3 \mathbf{b}(5.1 \mathrm{mM})$ and (b) $(R)-3 \mathbf{b} \cdot 4 \mathbf{b}(1.0 \mathrm{mM})$. 
ESI-MS Spectrum of $(R)-3 b \cdot 4 b$

a) Experimental ESI-MS Spectrum of $[(R)-\mathbf{3 b} \cdot \mathbf{4} \mathbf{b}+2 \mathrm{H}]^{2+}$

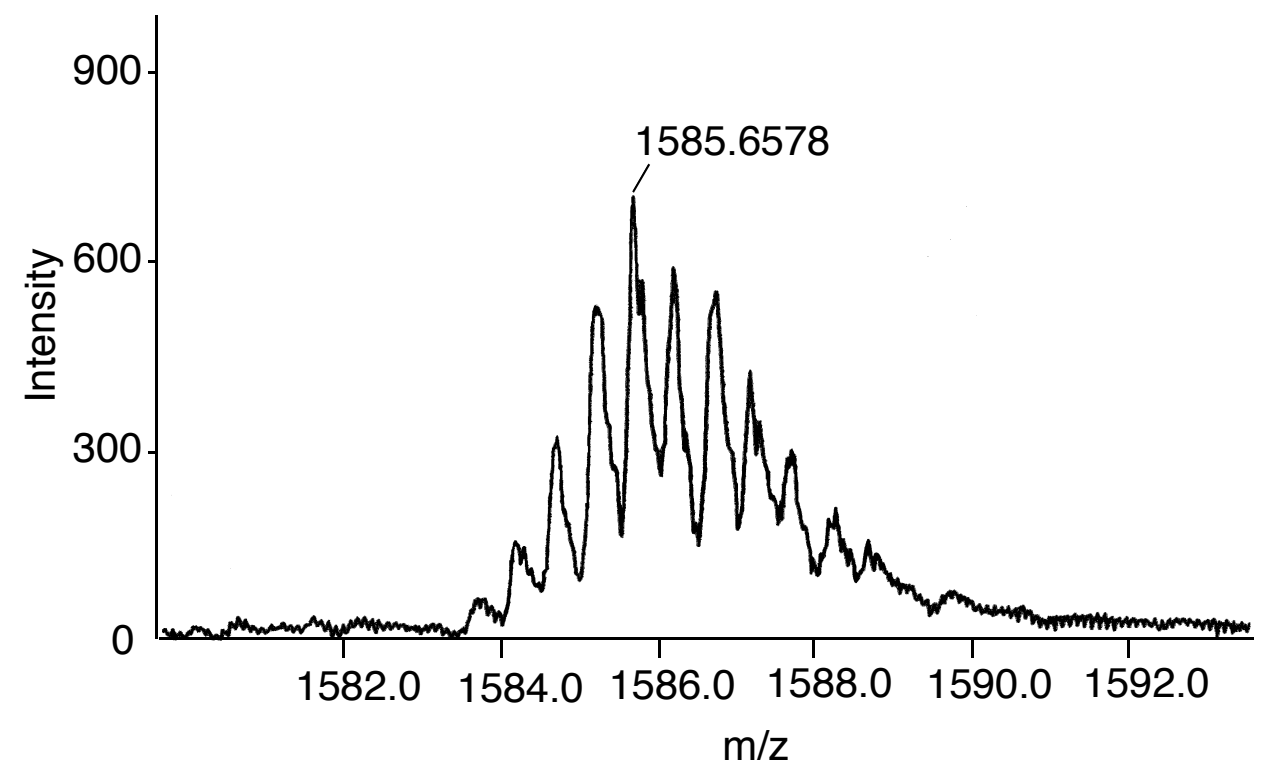

b) Isotope Simulation of $[(R)-3 \mathbf{b} \cdot \mathbf{4} \mathbf{b}+2 \mathrm{H}]^{2+}$

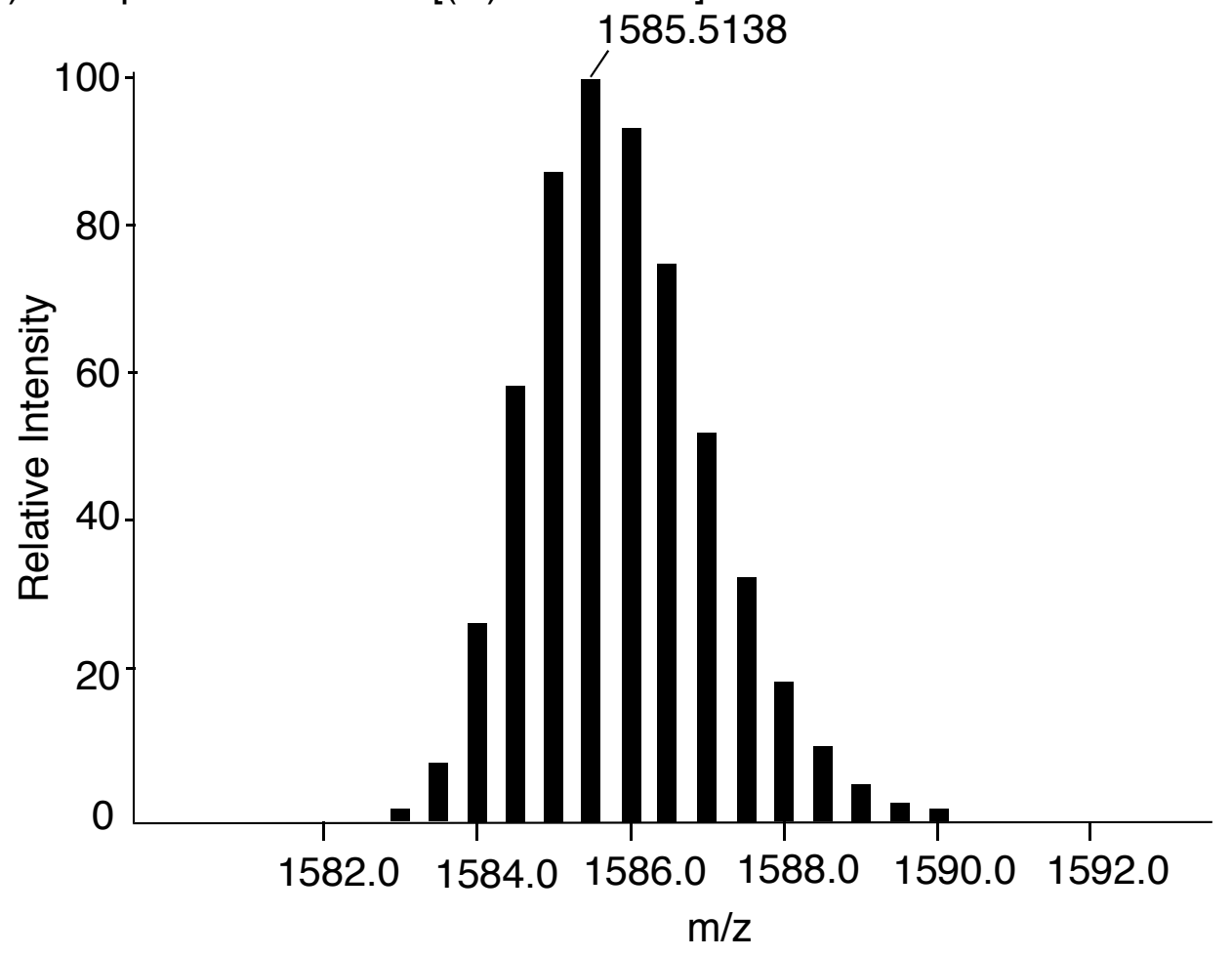

Figure S4. (a) Experimental and (b) simulated ESI mass spectra of $[(R)$ $3 \mathbf{b} \cdot 4 \mathbf{b}+2 \mathrm{H}]^{2+}$. 
6. ${ }^{31} \mathrm{P}$ NMR $\left(200 \mathrm{MHz}, \mathrm{CDCl}_{3}, 25{ }^{\circ} \mathrm{C}\right)$ Spectral Data

Table S1. ${ }^{31} \mathrm{P}$ NMR (200MHz, $\left.\mathrm{CDCl}_{3}\right)$ Spectral Data.

\begin{tabular}{cccccc} 
compound & $\delta / \mathrm{ppm}$ & $\mathcal{J}_{\mathrm{P}-\mathrm{Pt}} / \mathrm{Hz}$ & compound & $\delta / \mathrm{ppm}$ & $\mathcal{J}_{\mathrm{P}-\mathrm{Pt}} / \mathrm{Hz}$ \\
\hline$(R)-\mathbf{3 a}$ & 18.80 & 2627 & $(R)-\mathbf{3 b}$ & 52.71 & 2277 \\
$(S)-\mathbf{3 a}$ & 18.78 & 2627 & $(S)-\mathbf{3 b}$ & 52.72 & 2277 \\
$\mathbf{4 a}$ & 18.84 & 2641 & $\mathbf{4 b}$ & 52.60 & 2300 \\
$(R)-\mathbf{3 a} \cdot \mathbf{4 a}$ & 19.28 & 2650 & $(R)-\mathbf{3 b} \cdot \mathbf{4 b}$ & 52.92 & 2264 \\
& 18.52 & 2662 & & 51.92 & 2258 \\
$(S)-\mathbf{3 a} \cdot \mathbf{4 a}$ & 19.23 & 2672 & $(S)-\mathbf{3 b} \cdot \mathbf{4 b}$ & 52.92 & 2262 \\
& 18.51 & 2674 & & 51.93 & 2257 \\
\hline
\end{tabular}




\section{Optical Rotations}

Table S2. Optical Rotations.

\begin{tabular}{cccc} 
Compound & {$[\alpha]_{\triangleright}{ }^{25}$} & Compound & {$[\alpha]_{D}^{25}$} \\
\hline$(R)-\mathbf{3 a}$ & $-132\left(c 0.1, \mathrm{CHCl}_{3}\right)$ & $(R)-\mathbf{3 b}$ & $-105\left(c 0.1, \mathrm{CHCl}_{3}\right)$ \\
$(S)-\mathbf{3 a}$ & $+133\left(c 0.1, \mathrm{CHCl}_{3}\right)$ & $(S)-\mathbf{3 b}$ & $+102\left(c 0.1, \mathrm{CHCl}_{3}\right)$ \\
$(R)-\mathbf{3 a} \cdot \mathbf{4 a}$ & $-169\left(c 0.06, \mathrm{CHCl}_{3}\right)$ & $(R)-\mathbf{3 b} \cdot \mathbf{4 b}$ & $-249\left(c 0.06, \mathrm{CHCl}_{3}\right)$ \\
$(S)-\mathbf{3 a} \cdot \mathbf{4 a}$ & $+175\left(c 0.06, \mathrm{CHCl}_{3}\right)$ & $(S)-\mathbf{3 b} \cdot \mathbf{4 b}$ & $+255\left(c 0.06, \mathrm{CHCl}_{3}\right)$ \\
\hline
\end{tabular}




\section{Structural Transformation of the Duplexes}

$(\boldsymbol{R})-3 \mathbf{3} \cdot \mathbf{4 a}$ to $(\boldsymbol{R})-\mathbf{3 b} \cdot \mathbf{4 b}$. To a solution of $(R)-\mathbf{3 a} \cdot \mathbf{4 a}(3.4 \mathrm{mg}, 0.0010 \mathrm{mmol})$ in $\mathrm{CDCl}_{3}$ $(970 \mu \mathrm{L})$ was added a $0.1 \mathrm{M}$ solution of dppee in $\mathrm{CDCl}_{3}(22 \mu \mathrm{L}, 0.0022 \mathrm{mmol})$ at room temperature, and the reaction progress was monitored by ${ }^{1} \mathrm{H}$ NMR and CD.
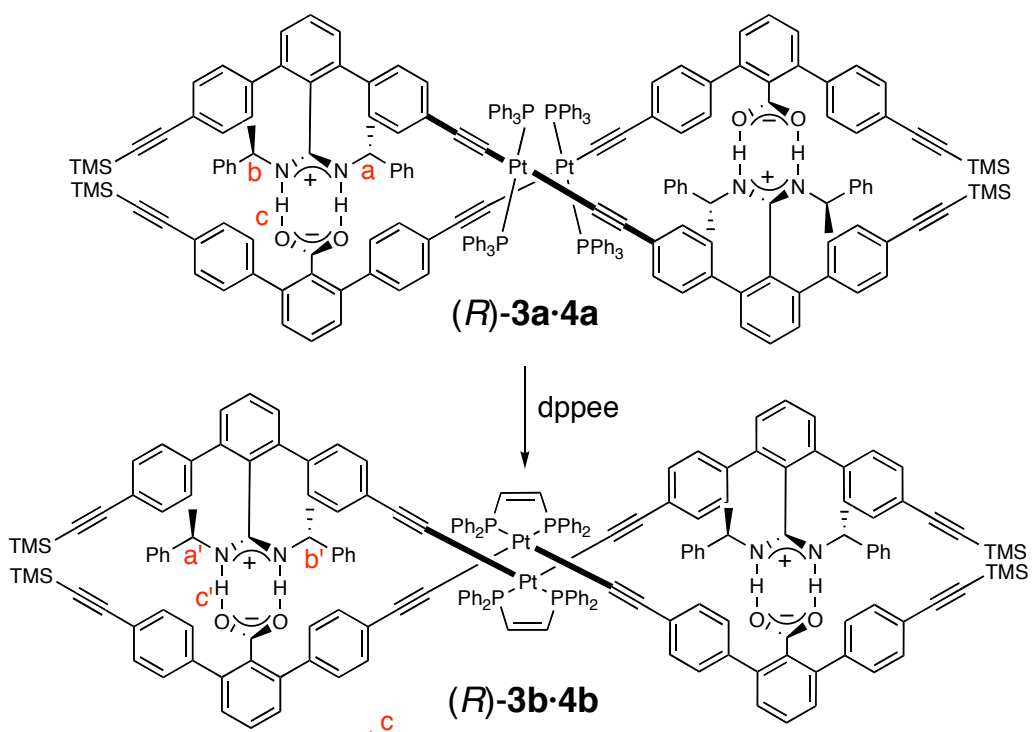

(a)

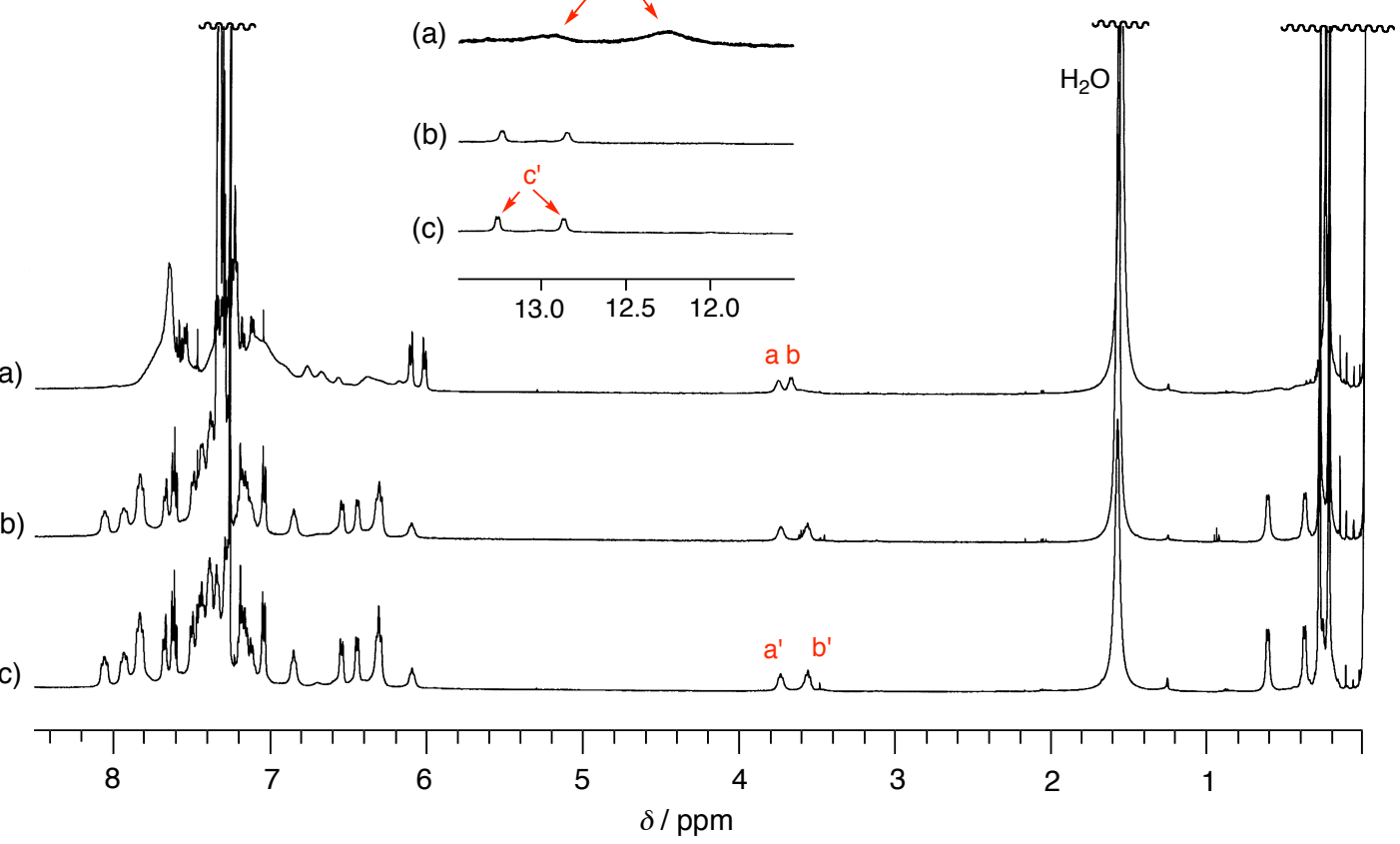

Figure S5. $\quad{ }^{1} \mathrm{H} \mathrm{NMR}\left(500 \mathrm{MHz}, \mathrm{CDCl}_{3}, 25{ }^{\circ} \mathrm{C}\right)$ spectra of $(\mathrm{a})(R)-\mathbf{3 a} \cdot \mathbf{4 a}(0.1 \mathrm{mM})$ and (c) $(R)-3 \mathbf{b} \cdot 4 \mathbf{b}(1.0 \mathrm{mM})$, and (b) a solution of $(R)-\mathbf{3 a} \cdot 4 \mathbf{a}(1.0 \mathrm{mM})+$ dppee $(2.2 \mathrm{mM})$ in $\mathrm{CDCl}_{3}$ after $2 \mathrm{~h}$ at ambient temperature. 

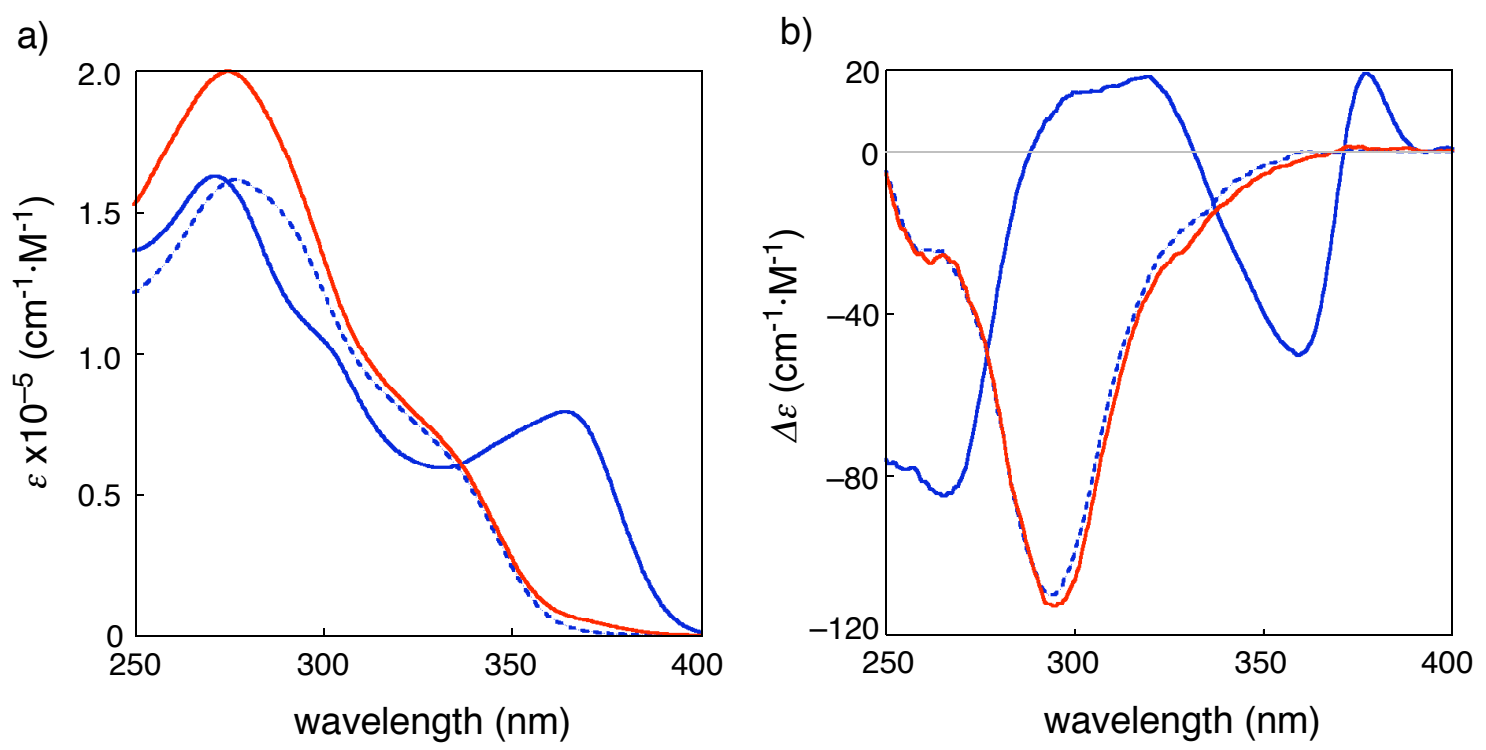

Figure S6. (a) UV/Vis and (b) CD spectra $\left(25^{\circ} \mathrm{C}\right)$ of $(R)-\mathbf{3 a} \cdot 4 \mathbf{a}(0.1 \mathrm{mM}$, blue solid line), $(R)-\mathbf{3 b} \cdot \mathbf{4 b}(0.1 \mathrm{mM}$, blue dashed line $)$, and a solution of $(R)-\mathbf{3 a} \cdot 4 \mathbf{a}(0.1 \mathrm{mM})$ and dppee $(0.22 \mathrm{mM})$ (red solid line) in $\mathrm{CDCl}_{3}$ after $2 \mathrm{~h}$ of mixing. 
Kinetics. The kinetics of the ligand exchange reactions of $\mathbf{3 a}, \mathbf{4 a}$, and $\mathbf{3 a} \cdot \mathbf{4 a}$ with dppee was investigated by using absorption spectroscopy. The exchange reactions obey the following equation.

$$
\mathrm{d}\left[\mathrm{Pt}-\mathrm{PPh}_{3}\right] / \mathrm{dt}=-\mathrm{k}\left[\mathrm{Pt}-\mathrm{PPh}_{3}\right][\mathrm{dppee}]
$$

where $\left[\mathrm{Pt}-\mathrm{PPh}_{3}\right]=[\mathbf{3 a}],[\mathbf{4 a}]$, or $[\mathbf{3 a} \cdot \mathbf{4 a}]$ and $\mathrm{k}$ is the second-order rate constant for the exchange reaction. Under the conditions in which equimolar amounts of the $\mathrm{PPh}_{3}$ complexes and dppee are employed, eq. 1 becomes

$$
\mathrm{dx} / \mathrm{dt}=-\mathrm{kx}^{2}
$$

Integration of eq. 2 gives

$$
1 / \mathrm{x}=1 / \mathrm{x}_{0}+\mathrm{kt}
$$

where $\mathrm{x}_{0}$ is the initial concentrations of the $\mathrm{PPh}_{3}$ complexes.

By using eq. 3 , the absorbance at $364 \mathrm{~nm}$ is given as follows:

$$
\mathrm{A}_{364}=\mathrm{c}_{1} \mathrm{x} /\left(1+\mathrm{x}_{0} \mathrm{kt}\right)+\mathrm{c}_{2}
$$

The reaction rates were determined from the least-squares curve fitting based on the eq. 4.

(a) $(R)-\mathbf{3 a}$ to $(R)-\mathbf{3 b}$

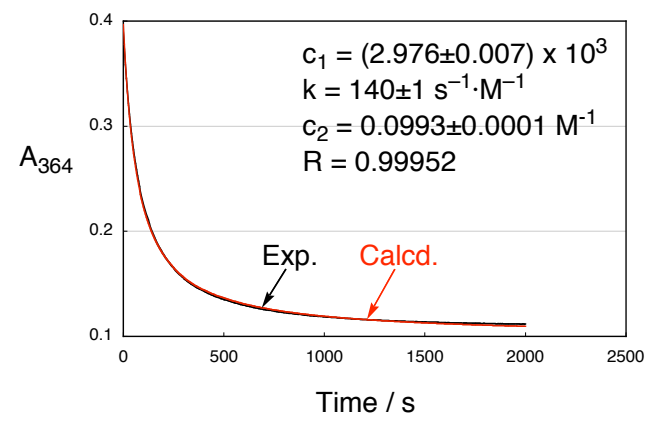

(c) $(R)-3 \mathbf{a} \cdot \mathbf{4} \mathbf{a}$ to $(R)-\mathbf{3 b} \cdot \mathbf{4 b}$

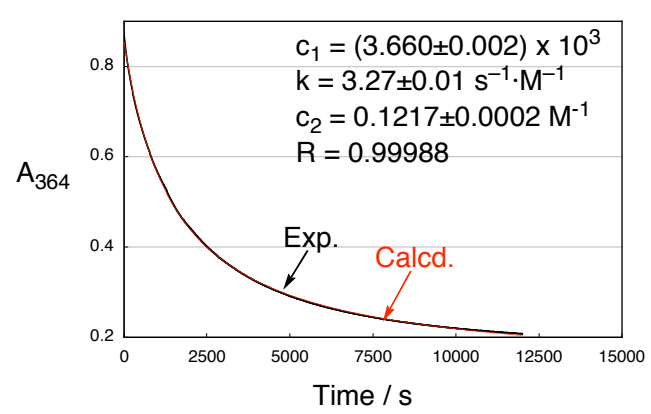

(b) $\mathbf{4 a}$ to $\mathbf{4 b}$

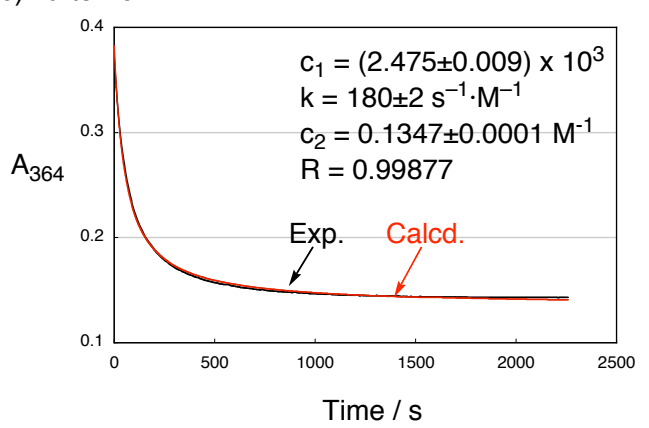

Figure S7. Plot of the absorbances at $364 \mathrm{~nm}$ against time for the ligand exchange reactions of (a) $(R)-\mathbf{3 a},(\mathrm{b}) \mathbf{4 a}$, and (c) $(R)-\mathbf{3 a} \cdot \mathbf{4 a}$ with dppee; $[(R)-\mathbf{3 a}]_{0}=[\mathbf{4 a}]_{0}=[(R)$ 3a $\cdot 4 a]_{0}=0.1 \mathrm{mM}$; cell length $=0.1 \mathrm{~cm} ; 25^{\circ} \mathrm{C}$. 
$(\boldsymbol{R})-\mathbf{3 b} \cdot \mathbf{4 b}$ to $(\boldsymbol{R})-\mathbf{1} \cdot \mathbf{2}$. To a solution of $(R)-\mathbf{3 b} \cdot \mathbf{4 b}(3.2 \mathrm{mg}, 0.0010 \mathrm{mmol})$ in $\mathrm{CDCl}_{3}$ $(960 \mu \mathrm{L})$ was added a $0.1 \mathrm{M}$ solution of $\mathrm{I}_{2}$ in $\mathrm{CDCl}_{3}(40 \mathrm{~mL}, 0.0040 \mathrm{mmol})$ at room temperature, and the reaction progress was monitored by ${ }^{1} \mathrm{H}$ NMR and CD.
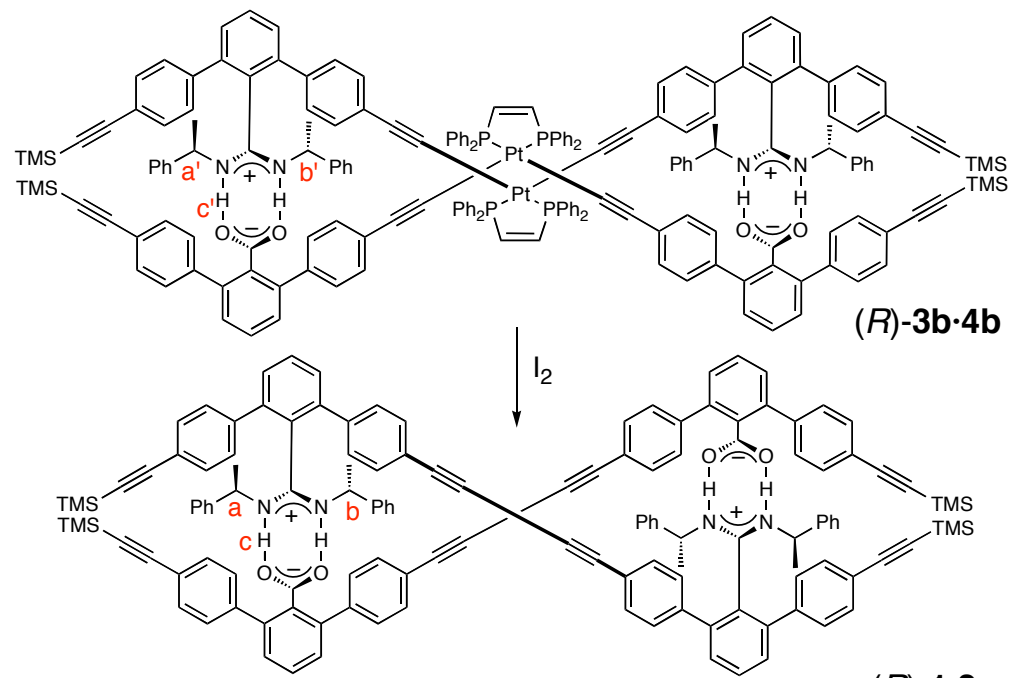

(a)

$(R)-1 \cdot 2$

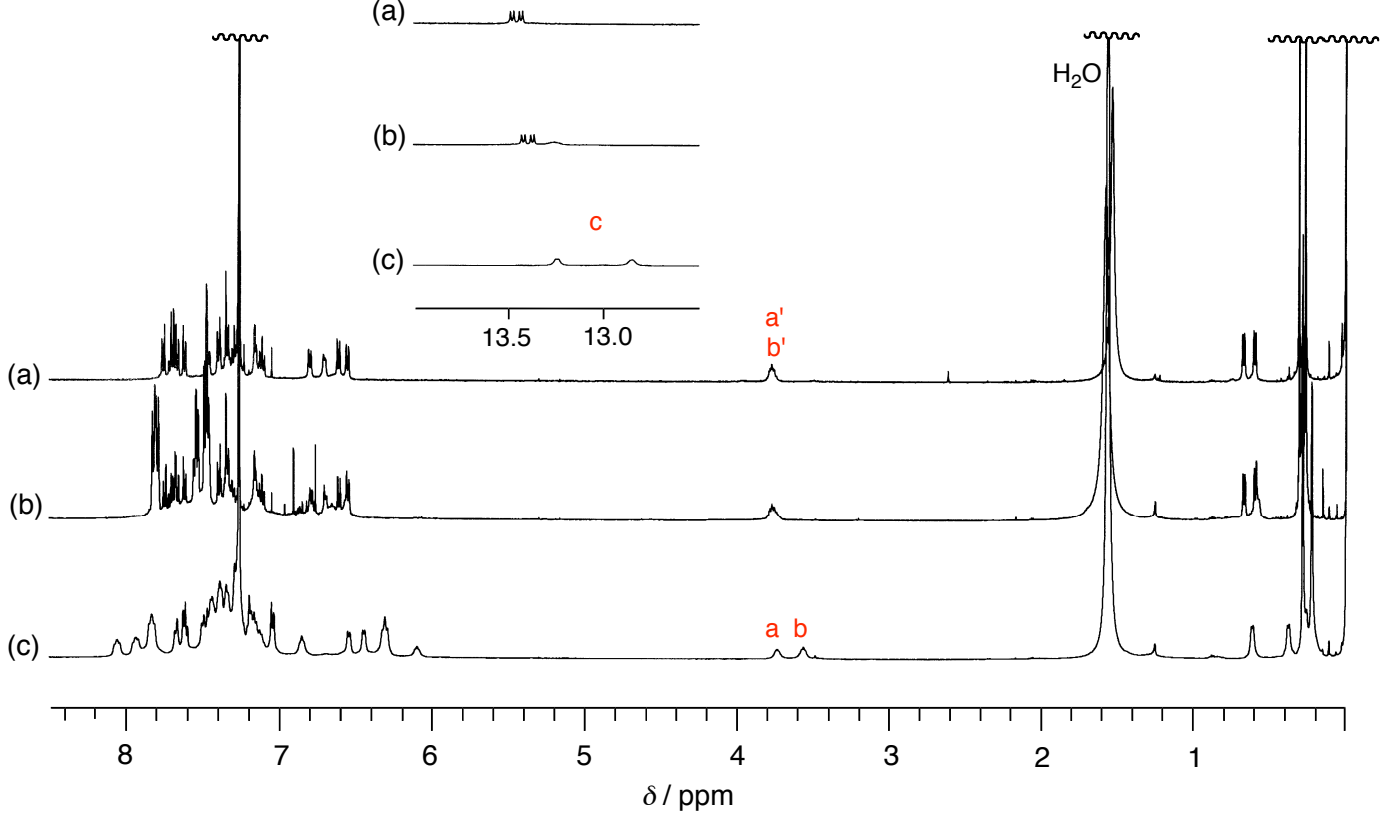

Figure S8. $\quad{ }^{1} \mathrm{H} \mathrm{NMR}\left(500 \mathrm{MHz}, \mathrm{CDCl}_{3}, 25^{\circ} \mathrm{C}\right)$ spectra of $(\mathrm{a})(R)-\mathbf{1} \cdot 2(0.1 \mathrm{mM})$ and (c) $(R)-3 \mathbf{b} \cdot 4 \mathbf{b}(1.0 \mathrm{mM})$, and (b) a solution of $(R)-3 \mathbf{b} \cdot 4 \mathbf{b}(1.0 \mathrm{mM})+\mathrm{I}_{2}(4.0 \mathrm{mM})$ in $\mathrm{CDCl}_{3}$ after $1 \mathrm{~h}$ of mixing. 

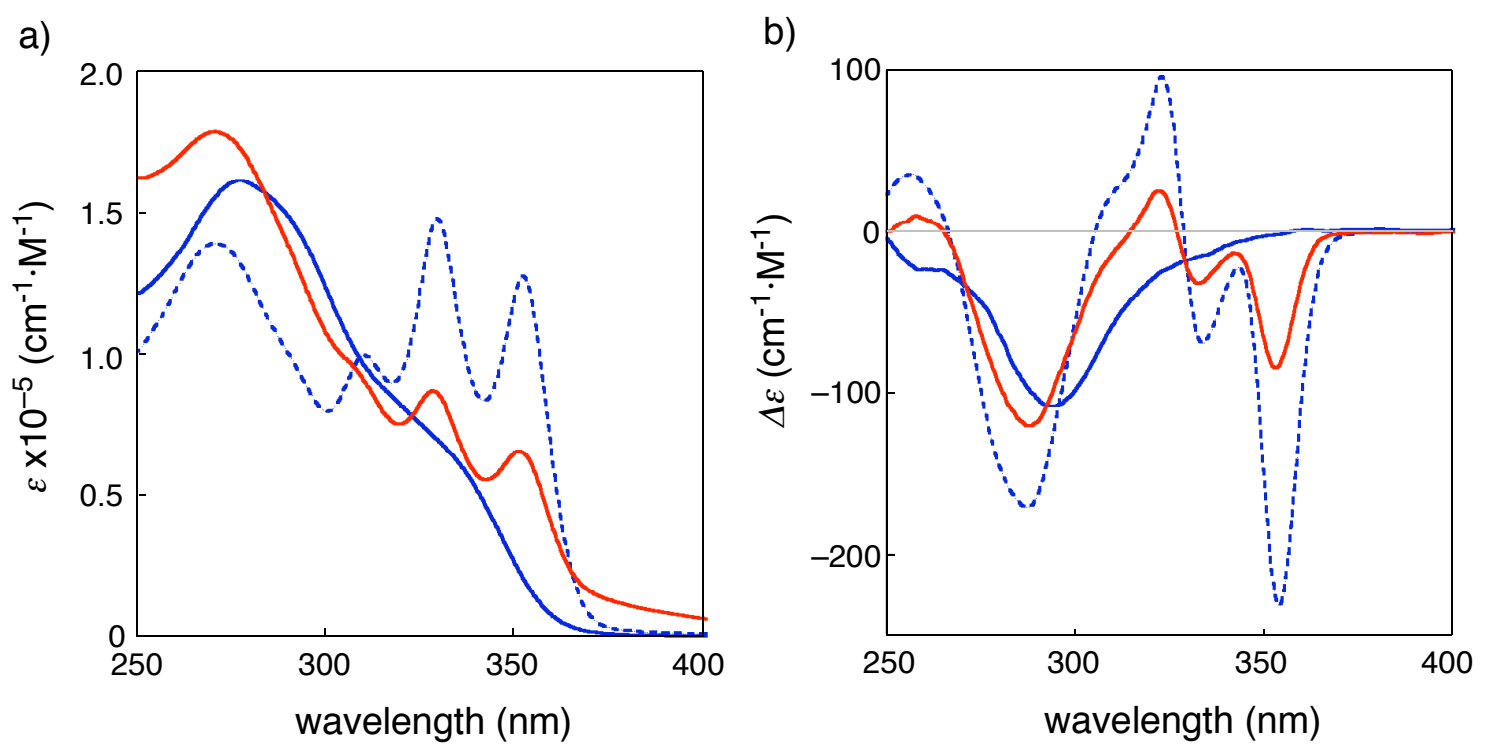

Figure S9. (a) UV/Vis and (b) CD spectra $\left(25^{\circ} \mathrm{C}\right)$ of $(R)-\mathbf{3 b} \cdot \mathbf{4 b}(0.1 \mathrm{mM}$, blue solid line) and $(R)-\mathbf{1} \cdot 2(0.1 \mathrm{mM}$, blue dashed line $)$, and a solution of $(R)-\mathbf{3 b} \cdot \mathbf{4 b}(0.1 \mathrm{mM})$ and $\mathrm{I}_{2}(0.40 \mathrm{mM})$ (red solid line) in $\mathrm{CDCl}_{3}$ after $2 \mathrm{~h}$ of mixing. 
9. MM-Calculated Structures of the Duplexes $3 a \cdot 4 a$ and $3 b \cdot 4 b$

(a)
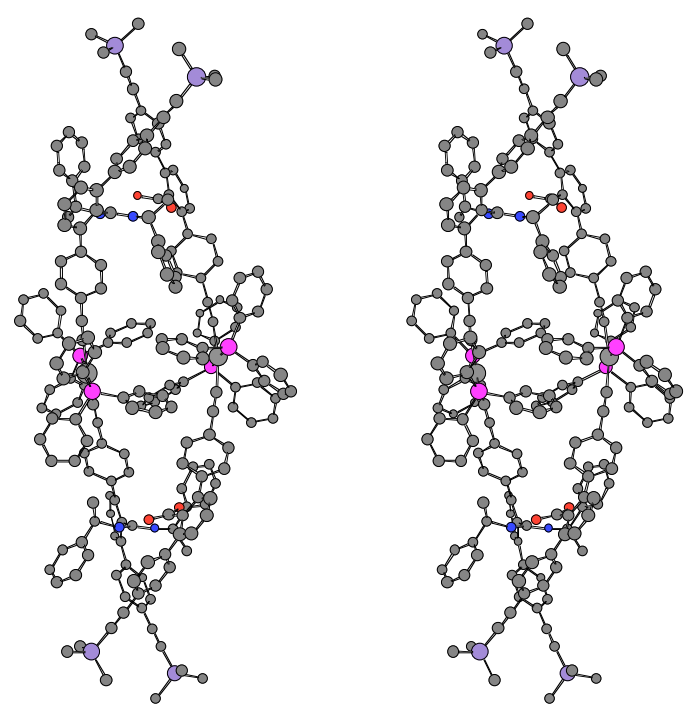

(b)
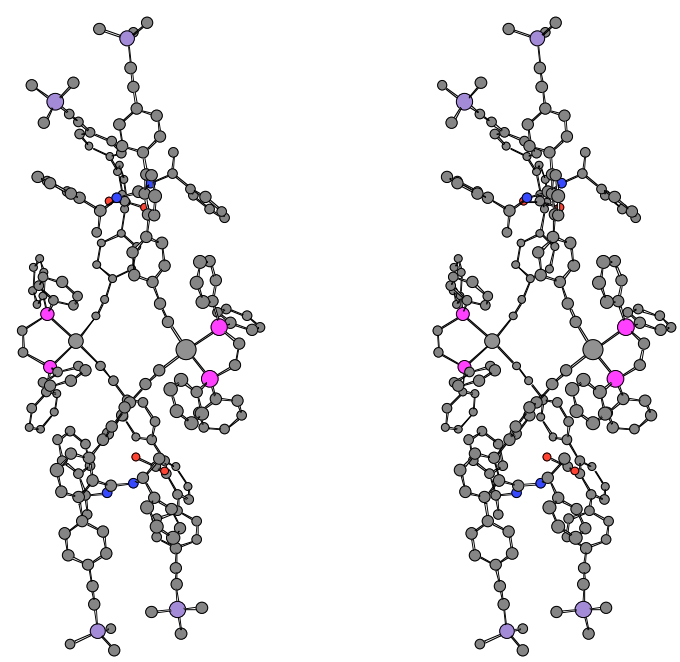

Figure S10. Stereoviews of MM-calculated structures of (a) $(R)-\mathbf{3 a} \cdot \mathbf{4 a}$ and (b) $(R)$ 3b·4b. Hydrogen atoms are omitted for clarity. Carbon (gray), nitrogen (blue), oxygen (red), silicon (purple), phosphorus (pink), and platinum (green). 